\title{
Influences of Cross-Sea High-Voltage Transmission Lines on Navigational
} Aids

\author{
Qinglong Hao, Jie Zhang*, Xueyuan Zhu \\ Dalian Maritime University, Dalian, China \\ *Corresponding Author.
}

\begin{abstract}
With the development of high-voltage electric power industry and the construction of cross-sea transmission projects in coastal ports, the interference of cross-sea high-voltage transmission lines on the performance of ship navigation aids gains considerable attention. The navigation safety control measures for ships crossing the waters of high-voltage transmission lines are proposed to study the navigational safety of the waters for the construction of high-voltage transmission lines across the sea. Conclusions with certain reliability are also drawn based on the analysis of the reasons and mechanisms of the interference phenomenon for the reference of maritime and scientific researchers. The images or signals of interference under the electromagnetic radiation from maritime shore-based radars, shipborne radars, shipborne VHF and GPS equipment through high-voltage transmission lines are obtained through on-site and actual ship testing.
\end{abstract}

Keywords: Cross-sea high-voltage transmission lines, Radar, Navigational aids, Interference

\section{Introduction}

High -voltage transmission lines have become the main power transmission methods due to their low investment costs, short construction periods and easy repair and maintenance. However, the energised high-voltage transmission line will emit electromagnetic waves to the surroundings as a radiation source. Particularly, the crosssea high-voltage transmission lines will interfere with the performance of shore-based or shipboard radars in use near the water. Such interference can adversely affect the navigation safety of ships. Although reports on maritime safety accidents are few due to such an interference, the electromagnetic interference generated by transmission lines is increasingly prominent. Electromagnetic interference has attracted the attention of maritime supervisors and ship pilots with the continuous construction of domestic high-voltage transmission lines across the sea.

Concerning the radar interference research, Qin Hongmin et al. obtained the magnetic field distribution and magnitude around high-voltage transmission lines through finite element analysis[1]. Liu Xingfa et al. gained the signal shadowing loss of high-voltage transmission lines to aviation radar stations through actual observation methods[2]. Yang Feng et al. performed ground-level radar image recognition on interference from high-voltage transmission lines through combined algorithms, such as inter-horizontal prediction filtering[3]. Gao Hongjun et al. conducted a comparative test on the ground static GPS interference under the interference of the high-voltage transmission line through actual measurement methods and obtained the interference value[4]. Qin Chaoqi calculated the interference value of the railway radio signal under the high-voltage power transmission line and obtained a certain conclusion[5]. Current domestic and foreign studies on the interference of overhead transmission lines are mostly analyses on the interference forms and measurements of transmission lines. The research on the cross-sea overhead transmission line often determines its safety from the design scale and layout scheme. However, the impact on maritime traffic is demonstrated by whether the clearance dimension meets the overhead clearance for navigation of ships, whilst the impact of overhead transmission lines on navigation aids, such as navigation radars, is not specifically studied[6]. At the beginning of 2019, the number of crossing sections of transmission lines in the navigable waters along the coast of Zhejiang, China alone, has reached 195. The designed

ISSN: 0010-8189

(C) CONVERTER 2020

www.converter-magazine.info 
overhead clearance heights of the 'Zhoushan and Mainland Interconnected Transmission Line (Luotou Waterway) Crossing Section' and the 'Zheneng Zhoushan Liuheng Power Plant Transmission Line (QinglongmenShuangyumen Channel) Crossing Section' located in Ningbo-Zhoushan Port are 70.5 and 59, m respectively. Both waterways are located above the important navigation channel of the core port area of Ningbo-Zhoushan Port. According to statistics, the number of navigable ships in the two spanning passages exceeded 100,000 (the position of the crossing segment is shown in Figure 1) in 2018. Once a traffic accident occurs, the channel will be blocked and a large number of ships will be delayed. Therefore, in the face of successive upgrades of high-voltage electrical pressurization systems in various regions in recent years, studying the impact of overhead transmission lines in a navigable waterway on the performance of shore-based and shipboard radar equipment is necessary.

The effect of high-voltage transmission lines across the sea is measured and researched from multiple angles of maritime shore-based radar, shipboard navigation radar, shipboard VHF and GPS equipment in this passage. The electromagnetic radiation interference generated by overhead transmission lines is effectively discriminated, and conclusions with certain credibility are drawn for reference to the maritime supervision and navigation practitioners.

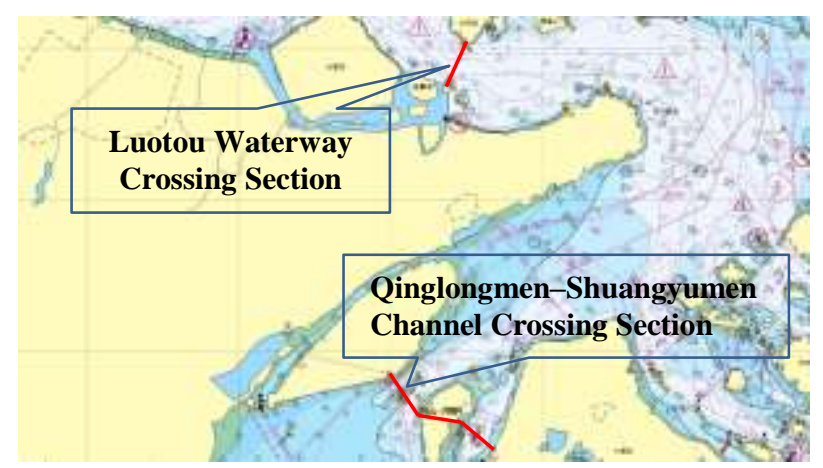

Figure 1: Schematic diagram of Luotou channel and Qinglongmen-Shuangyumen crossing section of high voltage transmission line

\section{Influences of high-voltage transmission lines on VTS shore-based radar}

VTS (Vessel Traffic Service) radar, which can monitor the navigation status of ships within the radar coverage area in real time, is an important maritime navigation aid for maritime supervision and traffic service. However, the monitoring of marine traffic by VTS radars will be limited to a certain extent due to the construction of highvoltage transmission lines across the sea. This construction is prominently manifested as overlapping interference of transmission line echoes across waters, especially when ships pass by near the transmission line. Such action reduces the capability of the VTS radar in distinguishing ships in the area, which affects the observations of passing ships by supervisors. The irregular echo image of Luotou and Qinglongmen-Shuangyumen channel crossing sections of high-voltage transmission line under the jurisdiction of Ningbo Maritime Safety Administration, which formed on the monitoring system of the VTS centre, is an example of such interference, as shown in Figure 2. The figure reveals that the image returned by the VTS radar across the high-voltage transmission line indicates a bright spot-like interference intermittently arranged along the direction of the power line, with unstable intensity and position of the bright spot.

ISSN: 0010-8189 


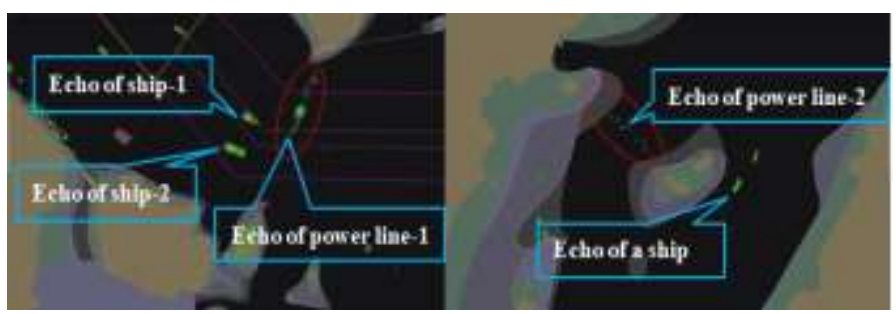

Figure 2: Radar images of the crossing section of Luotou waterway (power line 1) and the crossing section of Qinglongmen-Shuangyumen waterway (power line 2)

The bright speckled echo of the transmission line cannot be directly distinguished from the echo of a navigable ship physically; thus, this echo is easily mistaken for a ship as a false echo. However, if a ship only below the power line occurs at this time, then the real ship echo may be blocked or overlapped by the transmission line echo. This phenomenon leads to the echo loss of the ship or the exchange of objects, as shown in Figure 3.

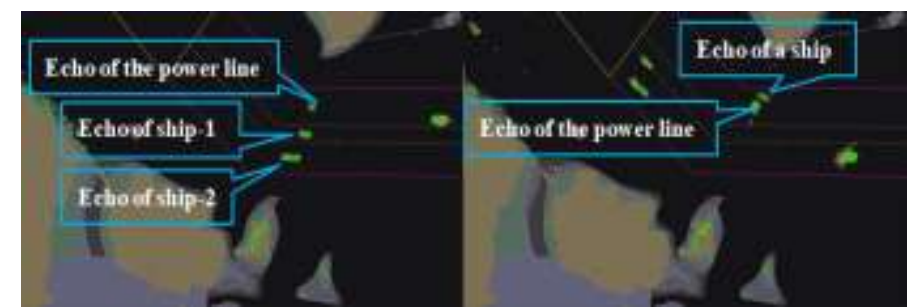

Figure 3: Radar images of power lines and ships across the section of Luotou channel

The actual measurement results show that the ship and the transmission line interference echoes observed at the VTS centre are similar in shape and difficult to be distinguished. If a ship only below the power line occurs at this time, then the real ship echo may be blocked or overlapped by the transmission line echo, leading to the loss or the exchange of objects. The ship's echo is easily distinguished when the ship trajectory is observed. However, when the electric cable swings due to weather conditions, such as wind and other factors, the transmission line interference echo is unstable, and the intensity and position of the interference bright spots are not fixed. Thus, the transmission line interference echo also generates an active track. The radar image of the ship is easily identified when the radar image is superimposed with the automatic identification system (AIS) signal of the ship, as shown in Figure 4. Navigable ships can show ship symbols and bow vector lines, whereas transmission line interference echoes do not possess the above characteristics. However, Class B ships that do not install the onboard AIS system and those that have closed the AIS system due to human factors also have no ship symbol and bow vector line[7].

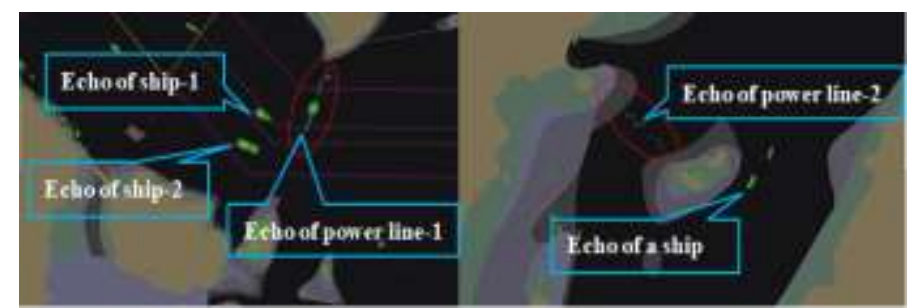

Figure 4: Radar images of ships AIS signals superimposed with the transmission line across the section of Luotou channel

Therefore, the maritime supervision department must strengthen the corresponding navigation management measures in the navigable waters with the high-voltage transmission line crossing and improve the VTS monitoring level in the jurisdiction. Supervisors should accumulate observation experience, increase radar observation methods (such as enabling ship backtrace display, superimposing AIS signals and opening radar automatic plotting) and familiarise themselves with the similarities and differences between transmission line interference

ISSN: 0010-8189

(C) CONVERTER 2020

Www.converter-magazine.info 
and ship echoes. They should also maintain close ties with passing ships and strengthen reminders on the safety of ships on transmission lines, especially under poor visibility weather. Moreover, supervisors should prohibit vessels from fishing, anchoring or staying for long periods under transmission lines to ensure the efficiency and safety of maritime traffic for vessels crossing waters. When necessary, a CCTV monitoring system should be added at an appropriate position under the high-voltage transmission lines across the sea to assist in the supervision of the waters across the section[8].

\section{Influences of high-voltage transmission lines on shipboard radars}

Shipboard radars are mainly used for navigation avoidance, ship positioning and pilotage in narrow waterways. These radars provide necessary lookout means for sailors, especially when visibility is poor. Radar performance, which is an indispensable tool for modern waterway traffic, directly affects the safety of ships and port traffic. The electromagnetic wave transceiver mechanism of shipboard radar will be theoretically interfered with by the electromagnetic radiation of high-voltage transmission lines. Thus, further studying and discriminating shipboard radar images crossing the high-voltage transmission line and the electromagnetic radiation interference is necessary.

Real-ship radar image tests of sea cruise ships are performed on the high-voltage transmission lines across the Qinglongmen-Shuangyumen channel to test the impact of high-voltage transmission lines on shipboard radar images. The weather conditions at testing were suitable, the visibility was good, the radar wave clutter was small and the echo was clear. The experimental ship departed from the Meishan Maritime Patrol Pier and traversed the under-sea high-voltage transmission line Qinglongmen at the actual measurement site. The changes in the shipboard radar echo and the interference image are continuously observed during the experiment, and the distance between the high-voltage line echo and the experimental ship is recorded. After the experiment is started, the highvoltage transmission lines were observed as continuous bright spots on the radar screen when the experimental ship departed and gradually approached the span, and the bright spots became clear as the ship approached, as shown in Figure 5. When the experimental ship approached the crossing section, the echo bright spots became blurred again and generally disappeared when the ship sailed directly below the section. After leaving the section, the echo bright spots gradually became clear again, as shown in Figure 6. When the experimental ship departed for a certain distance from the section, the echo bright spots were gradually blurred until their disappearance with the increase in the radar action distance, as shown in Figure 7.

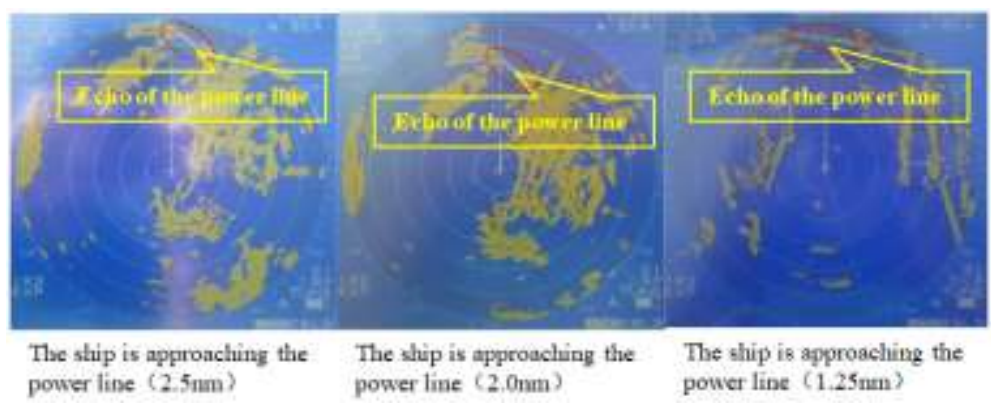

Figure 5: Radar image of the experimental ship approaching the power line 


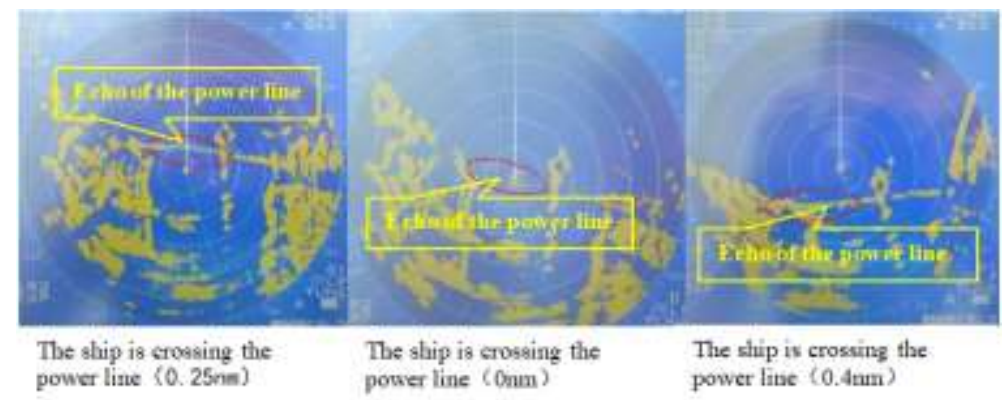

Figure 6: Radar image of the experimental ship during crossing

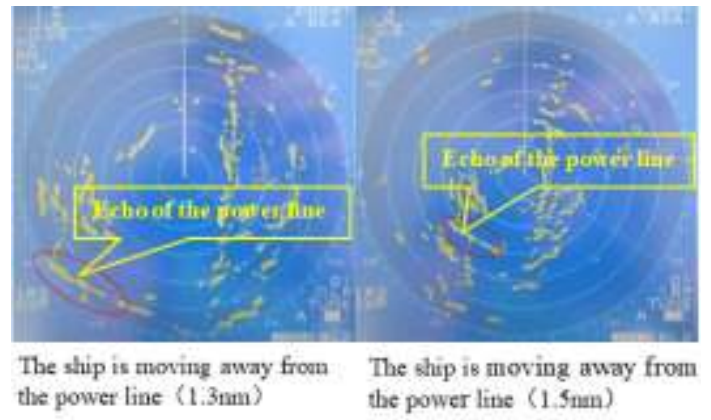

Figure 7: Radar image of the experimental ship moving away from the power line

The comparative analysis shows that during the entire voyage, the strength of the interference echo of the highvoltage transmission line is related to the distance between the radar antenna and the high-voltage transmission line. Within a certain range, the interference echo of the high-voltage transmission line is gradually increased as the ship approaches. However, when the ship approaches the transmission line sufficiently close, the interference echo of the transmission line will be gradually weakened until its disappearance when the ship is directly below the transmission line. Similarly, when the ship leaves the high-voltage transmission line at a short distance, the interference echo of the power line is gradually increased; when the ship continues to leave, the interference echo of the power line will be gradually weakened until its disappearance. By contrast, as the experimental ship approached the transmission line, the distance of the radar to the transmission line is gradually decreased, whilst the incident angle of the radar antenna is gradually increased. Combined with the experimental phenomena, the radar interference echo of the high-voltage transmission line is affected by the incident angle of the radar wave and its working distance. When the ship travels from far to near, the combined effect of the radar distance and the incident angle reaches a maximum value at a certain location. Thus, the interference echo of the transmission line also reaches a maximum value. When the ship continues to approach the transmission line, the incident angle of the radar wave is considerably increased, and the longitudinal component of the radar transmitted wave reaches a minimum value when the incident angle reaches $90^{\circ}$. Therefore, the received interference echo also reaches a minimum value. However, during the course of the experimental ship crossing the high-voltage transmission line waters, the internal noise, sea wave interference and co-frequency interference displayed on the radar screen are slightly changed, and other radars perform as usual.

The above actual measurement results show that the cross-sea high-voltage transmission line can interfere with the shipboard radar equipment in a certain range. When the distance between the transmission line and the ship is less than the maximum interference distance of the transmission line, the interference echo of the high-voltage line will be displayed on the radar screen. Notably, if other objects directly pass below the transmission line, then the echo of the object may be blocked by the interference echo of the transmission line. This blockage affects the radar observation of the ship's target. If echoes from multiple nearby targets simultaneously appear on the radar display, then the radar may be subject to target exchange due to interference from the transmission line, and the planned tracking targets may be lost. All these conditions elicit sufficient vigilance and attention of navigators.

ISSN: 0010-8189

(C) CONVERTER 2020

www.converter-magazine.info 
Therefore, when a ship sails in waters with high-voltage transmission lines across the sea, navigators should focus on the changes in radar images and properly adjust radar gain and interference suppression to reach the optimal status of the echo. Meanwhile, carefully distinguishing the interference echo from the target echo and avoid mistakenly considering the interference of the transmission line as the target echo is necessary. Moreover, strengthening the lookout and communication methods and focusing on the ships under the transmission lines is crucial to prevent misjudgement caused by interference and target echoes. Furthermore, from the viewpoint of safety, the ship should attempt to avoid fishing, anchoring or staying under such sections for a long time. The construction ship should work under the crossing section after obtaining the consent of traffic management.

\section{Influences of high-voltage transmission lines on maritime shipboard VHF communication}

Very high frequency (VHF) is the most commonly used on-site radio communication method for water transportation, which is crucial in ensuring the safety of ships. Considering that the high-voltage transmission line will theoretically exert radio interference to mobile communication signals, marine VHF equipment must be used to investigate the impact of the cross-sea high-voltage transmission line. Studies have shown that the electromagnetic radiation interference of high-voltage transmission lines mainly affects telecommunication signals below the VHF band (less than $30 \mathrm{MHz}$ ), whilst the interference of high-voltage transmission lines is rapidly decayed with the increase in frequency[9]. The electromagnetic radiation interference will not affect the telecommunication signal at more than $10 \mathrm{~m}$ away from the transmission line[10]. Although the marine VHF signal frequency is mainly concentrated around $160 \mathrm{MHz}(156-174 \mathrm{MHz})[11]$, which is far larger than $30 \mathrm{MHz}$, and the distance between the cross-sea high-voltage transmission line and the VHF transceiver antenna of the ship is more than $10 \mathrm{~m}$, the VHF communication effect is unaffected.

The researchers conducted real ship tests under the two high-voltage transmission line crossing sections in this study to verify the above conclusions experimentally. Ship-to-ship and ship-to-shore VHF communications were conducted several times at different angles. No effective interference affecting the communication effect was found from the senses, and the signal was clear without delay. Therefore, the influence of electromagnetic interference on the cross-sea high-voltage transmission lines on marine VHF communications is negligible.

\section{Influences of high-voltage transmission lines on maritime shipboard GPS}

Considering the principle of shipboard GPS positioning and navigation wireless signal transmission, studying the impact of high-voltage transmission lines on shipboard GPS positioning accuracy is also necessary. The transmission of GPS signals on high-voltage transmission lines is inevitably affected by electromagnetic fields. The transmission effects are as follows: firstly, the error in the pseudo-range measurement is increased and the positioning accuracy is reduced; secondly, the GPS signal is interfered, thus influencing the satellite acquisition and tracking of the GPS receiver[12]. Professor Ming Zutao conducted measurement experiments in Wujiawan, Wuhan[8], and the experimental results are as follows. The maximum GPS positioning error under a high-voltage transmission line appears directly below the high-voltage transmission line. Meanwhile, the maximum error of GPS range occurs when the measurement line is parallel to and passes through the high-voltage line. Both errors do not exceed $0.2 \%$. Therefore, GPS technology is fully capable of positioning and navigation with low accuracy requirements.

The researchers continuously recorded the position of the experimental ship under the high-voltage transmission line across the sea at a period of $10 \mathrm{~s}$ during the experiment with the ship to verify the above conclusions. Comparisons revealed that the GPS ship position is of good continuity before, during and after crossing. The GPS route is also of a high consistency with the actual sailing route and course without evident deviation. Therefore, the influence of the high-voltage transmission line across the sea on the navigation accuracy of the marine GPS can be ignored.

ISSN: 0010-8189

(C) CONVERTER 2020

Www.converter-magazine.info 


\section{Conclusion}

The above research and analysis show that with the occurrence of a certain form of electromagnetic radiation interference around the cross-sea high-voltage transmission line, the performance of ship navigation aids, such as maritime shore-based and shipboard radars, can be affected. The interference generated by the high-voltage transmission lines on the radar forms false echoes on the screen. These false echoes easily lead to erroneous identification or tracking failure of the target by maritime supervision or navigation practitioners, posing a remarkable impact on the performance of the equipment. Therefore, radar users must be vigilant and carefully judge the interference of high-voltage transmission lines by various means to ensure navigation safety. Moreover, ships should be prevented from crossing the sea high-voltage transmission lines for fishing, anchoring or staying for a long time. The influences of high-voltage transmission lines on maritime shipboard VHF communication and GPS positioning navigation can be ignored.

\section{Acknowledgment}

This paper was funded by the Fundamental Research Funds for the Central Universities of China (Grant No. 3132020143).

\section{References}

[1] H. Qin, W. Cheng, H. Qin, et al, "Analysis of Magnetic Field of High-Voltage Overhead Transmission Lines,” Telecom Power Technology, vol. 33, no. 3, pp. 125-127, 2016.

[2] X. Liu, Z. Gan, N. Li, et al, "Analysis and Experimental Research on Electromagnetic Effect of UHV Transmission Lines to Air Defense Surveillance Radar Station Nearby,” High Voltage Engineering, vol. 44, no. 10, pp. 3299-3306, 2018.

[3] F. Yang, y. Gao, W. Tang W, "Identification and Removing of the City High Voltage Interference From Geological Radar Profile,” Chinese Journal of Engineering Geophysics, vol. 2, no. 4, pp. 276-281, 2015.

[4] H. Gao, X. Feng, Y. Gao, "Testing and Analysis on the Influence of High Voltage Transmit Electrical Wire on Static GPS Measurement," Geomatics and Spatial Information Technology, vol. 41, no. 6, pp. 132-134, 2018.

[5] Z. Qin, "Study on the Electromagnetic Interference between High-voltage AC overhead Transmission Lines and Railway Communication Signal System," Ph.M. Thesis, Zhejiang University, China, 2018.

[6] Y. Zhao, Y. Li, H. Yan, "Study on the Influence of Aerial Transmission Lines in Navigable Watercourses on Navigation Radar. Marine Technology,” no. 6, pp. 31-33, 2012.

[7] Z. Gao, Y. Zhang, J. Liu, "Abnormal information judgment and analysis of AIS. Ship and Ocean Engineering,” vol. 64, no. 4, pp. 220-222, 2017.

[8] Y. Zheng, X. Cheng, L. Gan, "Shadowing Effect of Coastal Building on VTS Radar," Navigation of China, vol. 41, no. 7, pp. 1-6, 2018.

[9] D. Wei, "Analysis of Radio Interference on Mobile Telecommunication Station from HV Overhead Transmission Lines," Electric Power Construction, vol. 26, no. 7, pp. 22-24, 2005.

[10] S. Ma, "500kVTransmission line feedback of radio interference (Rl)," Power System Technology, no. 6, pp. 42-48, 2010.

[11] Z. Wang, H. Jin, M. Luo, et al. "Design of Marine adjustable inverted L VHF antenna," Ship and Ocean Engineering, vol. 48, no. 1, pp. 45-48, 2019.

[12] M. Yu, R. Xuan, "Research on ship navigation GPS positioning data processing," Ship and Ocean Engineering, vol. 37, no. 6, pp. 92-95, 2008. 